\title{
Isolation of Pristine Electronics Grade Semiconducting Carbon Nanotubes by Switching the Rigidity of the Wrapping Polymer Backbone on Demand
}

\author{
Yongho Joo, ${ }^{\dagger}$ Gerald J. Brady, ${ }^{\dagger}$ Matthew J. Shea, ${ }^{\dagger}$ M. Belén Oviedo, ${ }^{\ddagger}$ Catherine Kanimozhi, ${ }^{\dagger}$ \\ Samantha K. Schmitt, ${ }^{\dagger}$ Bryan M. Wong, ${ }^{\ddagger}$ Michael S. Arnold, ${ }^{\dagger}$ and Padma Gopalan ${ }^{*,+}$ \\ ${ }^{\dagger}$ Department of Materials Science and Engineering, University of Wisconsin—Madison, Madison, Wisconsin 53706, United States and ${ }^{\ddagger}$ Department of Chemical and \\ Environmental Engineering, and Materials Science and Engineering Program, University of California—Riverside, Riverside, California 92521, United States
}

\begin{abstract}
Conjugated polymers are among the most selective carbon nanotube sorting agents discovered and enable the isolation of ultrahigh purity semiconducting singled-walled carbon nanotubes (s-SWCNTs) from heterogeneous mixtures that contain problematic metallic nanotubes. The strong selectivity though highly desirable for sorting, also leads to irreversible adsorption of the polymer on the s-SWCNTs, limiting their electronic and optoelectronic properties. We demonstrate how changes in polymer backbone rigidity can trigger its release from the nanotube surface. To do so, we choose a model polymer, namely poly[(9,9dioctylfluorenyl-2,7-diyl)-alt-co-(6,60-(2,20-bipyridine))] (PF0-BPy), which provides

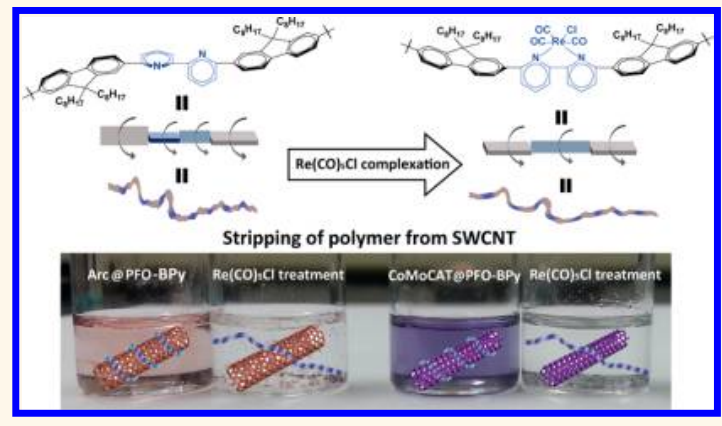
ultrahigh selectivity for s-SWCNTs, which are useful specifically for FETs, and has the chemical functionality (BPy) to alter the rigidity using mild chemistry. Upon addition of $\operatorname{Re}\left(\mathrm{CO}_{5}{ }_{5} \mathrm{Cl}\right.$ to the solution of PFO-BPy wrapped s-SWCNTs, selective chelation with the BPy unit in the copolymer leads to the unwrapping of PF0-BPy. UV-vis, XPS, and Raman spectroscopy studies show that binding of the metal ligand complex to BPy triggers up to $85 \%$ removal of the PFO-BPy from arc-discharge s-SWCNTs (diameter $=1.3-1.7 \mathrm{~nm}$ ) and up to $72 \%$ from CoMoCAT s-SWCNTs (diameter $=0.7-0.8 \mathrm{~nm}$ ). Importantly, Raman studies show that the electronic structure of the s-SWCNTs is preserved through this process. The generalizability of this method is demonstrated with two other transition metal salts. Molecular dynamics simulations support our experimental findings that the complexation of $\mathrm{BPy}$ with $\operatorname{Re}(\mathrm{CO})_{5} \mathrm{Cl}$ in the PFO-BPy backbone induces a dramatic conformational change that leads to a dynamic unwrapping of the polymer off the nanotube yielding pristine s-SWCNTs.
\end{abstract}

KEYWORDS: conjugated polymers $\cdot s$ - SWCNTs $\cdot$ polymer backbone rigidity

S ingle-walled carbon nanotubes (SWCNTs) have exceptional electronic properties that enable a multitude of semiconducting device applications such as field-effect transistors (FETs), ${ }^{1}$ photovoltaics, ${ }^{2}$ and gas sensors. ${ }^{3}$ To widely use SWCNTs as the semiconducting material in electronic devices, it is essential to separate the desirable semiconducting SWCNTs from as-synthesized electronically heterogeneous mixtures of metallic ( $\mathrm{m}$-) and semiconducting (s-) SWCNTs. The challenge of synthetic heterogeneity has motivated the development of a number of sorting techniques, including DNA separation, ${ }^{4}$ density gradient ultracentrifugation, ${ }^{5}$ surfactant assisted purification, ${ }^{6}$ chromatography, ${ }^{7}$ and polymer wrapping. ${ }^{8}$ Among these sorting methods, polymer wrapping is considered as one of the most effective methods for selectively isolating large quantities of electronically homogeneous s-SWCNTs. In particular, polyfluorene polymers have been studied as semiconducting-selective agents with selectivity for chirality, diameter and electronic type. ${ }^{8-10}$ Polyfluorenes enable a simple high-fidelity sorting process for milligram-scale quantities of s-SWCNTs to be used in high performance electronic devices and photovoltaic applications. ${ }^{11-13}$

Developing an understanding for the factors that lead to strong selective interactions between the conjugated polymer and the semiconducting tubes is evolving but *Address correspondence to
pgopalan@wisc.edu.

Received for review June 23, 2015 and accepted September 8, 2015.

Published online

10.1021/acsnano.5b03835

(c) XXXX American Chemical Society 
far from complete. The most common factors that seem to dominate are strong $\pi-\pi$ interactions of the conjugated polymer backbone to the tubes, which scales with tube diameter; ${ }^{8}$ the number of polymer repeat units that can wrap around a tube which correlates with the tube diameter and the rigidity of the polymer backbone; enantioselectivity of the polymer, ${ }^{13,14}$ and the length/sterics of the side chains. ${ }^{15}$ Most of these studies are based on the synthesis of a series of polymers with various structural characteristics and examining their effect on the sorting behavior and rationalizing the results using simulations. Recently, a tetrathiafulvalene vinylogue-fluorene copolymer was developed that changes conformation upon protonation with acid to reversibly wrap and unwrap SWCNTs. ${ }^{16}$ This is one of the very few examples of direct change in torsional angle of the polymer backbone to trigger the unwrapping process. Though quite effective in reversibly desorbing from the surface, the polymer exhibits strong selectivity only for smaller diameter tubes mainly those $\leq 1.0 \mathrm{~nm}$, and a detectable metallic background remains in the absorbance spectrum indicating poor electronic-type selectivity. There are other mechanisms by which polymer wrappers have been removed or degraded from the nanotubes that do not rely on changing the rigidity. For example: Wang et al. demonstrated separation of s-SWCNTs using degradable alternating copolymers, specifically poly[(9,9-dioctylfluorenyl-2,7-diyl)-alt-codisilane], which contains a disilane link that is degraded postsorting by a hydrofluoric acid treatment. ${ }^{17}$ However, the s-SWCNT sorting performance, as-measured by the metallic background in absorbance spectroscopy, is inferior to both PFO and PFO-BPy, and the use of $\mathrm{HF}$ is often not compatible with the device fabrication process. ${ }^{16,17}$ More recent studies have used an alternate approach based on supramolecular chemistry to assemble and disassemble a conjugated polymer in the presence of tubes. For example, the use of metalcomplexation driven ${ }^{18}$ or $\mathrm{H}$-bonding driven ${ }^{19}$ supramolecular assembly has been shown to reversibly disperse tubes, and following sorting, disassembly is triggered by addition of reagents to break the chelation or H-bonding, respectively. In the former approach, selectivity was shown more toward smaller diameter tubes, but removal of metallic tubes was not complete. In the latter approach carried out in organic solvents, selectivity toward larger diameter tubes was demonstrated with complete removal of metallic tubes. This latter approach is very promising yet synthetically intensive. Both these methods rely on reducing the chain length of the polymer to the oligomeric length scale to trigger the release.

We pick a commonly used commercially available polyfluorene derivative PFO-BPy as a model polymer to directly probe the effect of chain rigidity on the wrapping/unwrapping process on the s-SWCNTs.
Our choice of this model system is based on its commercial availability, outstanding sorting selectivity, availability of functional groups in the backbone to alter rigidity, and recent device results, which are described below. Recently, we demonstrated the extraordinarily electronic-type selectively of the polyfluorene derivative PFO-BPy by measuring the on/off ratio of FETs, where zero metallic nanotubes were encountered in the measurement of more than 5000 s-SWCNTs. ${ }^{9,10,20}$ Sonication of SWCNTs with dissolved polymer in organic solvents leads to PFO-BPy wrapped SWCNT (SWCNT@PFO-BPy) complexes that are soluble in solvents such as toluene and chloroform. Using the similar polymer poly(9,9-dioctylfluorene-2,7-diyl) (PFO), Bindl et al. demonstrated that even after several aggressive rinsing steps to remove free or excess polymer using ultracentrifugation, a significant amount of PFO remained bound to the SWCNT (nearly $50 \%$ by weight). ${ }^{21}$ These remaining polymer residues are expected to increase the contact resistance at the metal-nanotube interface of SWCNT FETs, ultimately limiting the conductance of these FETs at sub-100 nm channel lengths. ${ }^{22}$ This well-known limitation is inherent to solution-based SWCNT FETs, which also have larger device-to-device variation in contact resistance than directly grown CVD SWCNT FETs. ${ }^{17,22-24}$ A more complete removal of adsorbed polymer residues from the SWCNT surface is one possible approach toward improving contact resistance and reducing variation in device performance. ${ }^{17}$

Here, we demonstrate how the rigidity and $\pi-\pi$ interactions of a single polymer, namely PFO-BPy, can be tuned without additional synthesis to unwrap the polymer from s-SWCNTs. This approach retains the outstanding selectivity of PFO-BPy in the sorting process while enabling facile removal. We demonstrate an effective yet simple and mild method to remove postsorting, and the wrapping PFO-BPy copolymer from the surface of s-SWCNTs by switching the backbone rigidity. We use chelation chemistry to complex pentacarbonylrhenium chloride $\left(\operatorname{Re}(\mathrm{CO})_{5} \mathrm{Cl}\right)$ to the bipyridine (BPy) moiety in the wrapping copolymer backbone (Figure 1). We show the effectiveness of the chelation chemistry on both large and small diameter tubes. The chain-stiffness of the PFO-BPy changes upon complexation, likely providing the driving force to overcome the $\pi-\pi$ and electronic interactions ${ }^{8}$ which adhere the polymer to the nanotube. Optical absorbance, X-ray photoelectron spectroscopy (XPS), Raman spectroscopy, and large-scale molecular dynamics simulations were used to characterize the extent of PFO-BPy removal and configurational changes to the PFO-BPy after $\operatorname{Re}(\mathrm{CO})_{5} \mathrm{Cl}$ complexation (PFO-BPy:Re). The ability to switch the rigidity of the PFO-BPy in order to unwrap from the nanotubes opens up the door to new design strategies where specific functional groups can be placed on the 


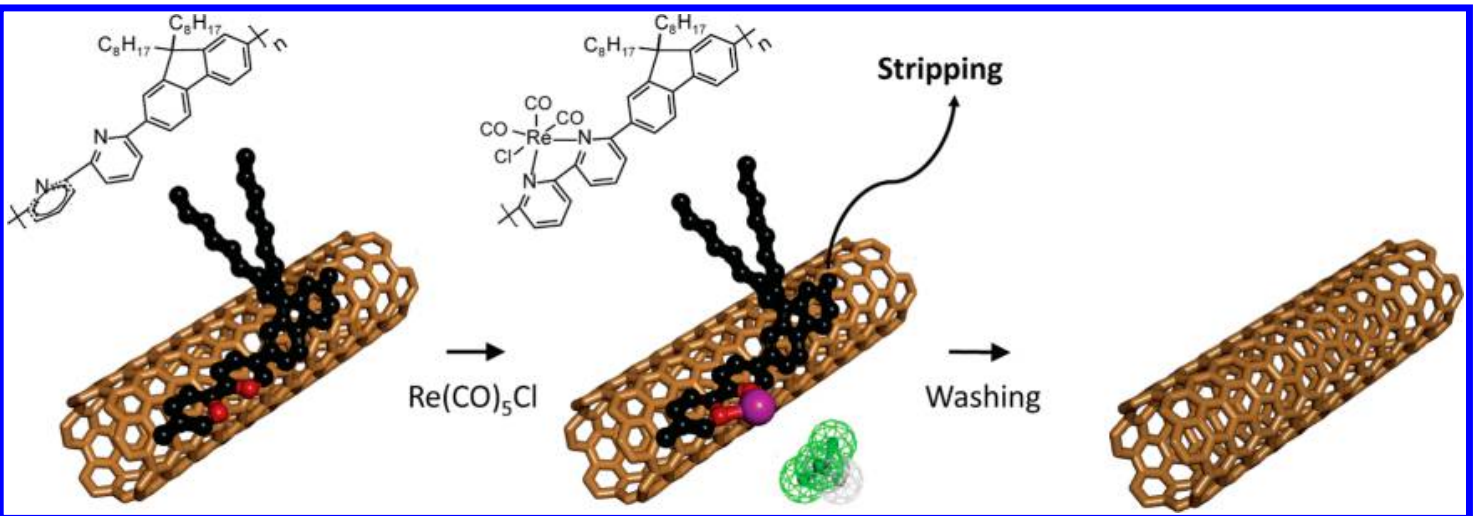

Figure 1. A schematic illustration of the process used to produce pristine s-SWCNTs by removing PFO-BPy from the SWCNT surface by complexation with pentacarbonylchlororhenium $(\mathrm{I})\left(\mathrm{Re}(\mathrm{CO}){ }_{5} \mathrm{Cl}\right)$. Carbon in PFO-BPy is represented by black, carbon in s-SWCNT by brown, nitrogen by red, rhenium by purple, and chlorine from chloroform solvent by green color.

polymer backbone to chemically alter the rigidity. This approach can potentially lead to new improved sorting strategies in which the concentration of problematic metallic nanotubes can be iteratively reduced. We envision that the removal of PFO-BPy can potentially be used to develop multistep sorting protocols where semiconducting tubes first isolated using PFO-BPy can then be subjected to an entirely different sorting protocol, for example, using a different conjugated polymer with orthogonal selectivity, aqueous surfactants for DGU, two-phase sorting, or even a second iteration of sorting using PFO-BPy. Ideally, our target is to use the best possible polymer to isolate exceptionally electronic-type monodisperse semiconducting nanotubes (i.e., PFO-BPy) and then remove and replace it with a designer polymer that can provide custom function (e.g., guide assembly into arrays, decompose on demand to improve the contact resistance, or drive charge separation or collection in photovoltaic devices).

\section{RESULTS AND DISCUSSION}

SWCNTs can be synthesized by a variety of methods, each producing a unique set of diameters and chiralities. SWCNTs synthesized using the Arc-discharge process (Arc) have diameters near $1.5 \mathrm{~nm}$ and band gaps of several hundred millielectronvolts. The cobaltmolybdenum catalyst process (CoMoCAT) produces small diameter nanotubes rich in $(6,5)$ chirality with diameters less than $1 \mathrm{~nm}$ and band gaps near $1.2 \mathrm{eV}$. Regardless of synthetic procedure, all nanotubes share specific optical and electronic properties due to the one-dimensional quantum confinement of electrons in the $\pi$-orbitals of the $\mathrm{sp}^{2}$ carbon atoms that make up the nanotube. Specifically, optical absorption spectroscopy reveals a series of sharp optical transitions known as $S_{1}$ (band gap), $S_{2}, S_{3}$, and so on, with each transition in the series a higher energy than the last. These optical transitions can be used to quantify the concentration of each nanotube in solution or film. The $\mathrm{S}_{2}$ and $\mathrm{S}_{3}$ transitions lie in the visible spectrum for
CoMoCAT-SWCNTs and Arc-SWCNTs, respectively, giving solutions of these nanotubes color and the optical absorptivity at these transitions provides a simple measure of the concentration of SWCNTs in solution. Dispersion of both CoMoCAT and Arc-SWCNTs with PFO-BPy followed by selective sedimentation and filtration protocols is known to yield homogeneous solutions of semiconducting nanotubes that are completely isolated, as confirmed by optical absorbance and photoluminescence spectroscopy. ${ }^{8}$

Here, $10 \mathrm{mg} / \mathrm{mL}$ of $\operatorname{Re}(\mathrm{CO})_{5} \mathrm{Cl}$ in chloroform was added to two different SWCNT solutions, the first containing PFO-BPy-sorted Arc-discharge SWCNTs (Arc@PFO-BPy) and the second containing PFO-BPy-sorted CoMoCAT SWCNTs (CoMoCAT@PFO-BPy). Both solutions contained $10 \mu \mathrm{g} / \mathrm{mL}$ of SWCNT and $20-40 \mu \mathrm{g} / \mathrm{mL}$ of PFO-BPy. A dramatic difference in the color of the solutions was observed. In both cases, the supernatant solution turned colorless and black precipitates were detected (Figure 2a). In contrast, no color change or precipitation was observed in control samples of nanotubes wrapped by PFO, as PFO does not have the chelating BPy units to complex with the transition metal. These control samples (HiPco@PFO) were prepared from nanotubes grown by the high-pressure carbon monoxide method (HiPco) with diameters ranging from 0.8 to $1.2 \mathrm{~nm}$. The nanotube@PFO-BPy complexes are typically known to be soluble for long durations in chloroform; ${ }^{20}$ therefore, we infer that it is the selective action of the $\operatorname{Re}(\mathrm{CO}){ }_{5} \mathrm{Cl}$ complexation to the PFO-BPy that causes the precipitates to appear.

The precipitated Arc@ and CoMoCAT@PFO-BPy:Re following complexation were collected by centrifuging the precipitates into a pellet (referred to as posttreatment tubes). These precipitates were then sequentially dispersed in THF, chloroform, and methanol and then centrifuged in order to remove free polymer and excess $\operatorname{Re}(\mathrm{CO})_{5} \mathrm{Cl}$ (referred to as postwash tubes). THF and chloroform are good solvents for the polymers, whereas methanol is a good solvent for $\operatorname{Re}(\mathrm{CO})_{5} \mathrm{Cl}$. The nanotubes at the different stages were dispersed 


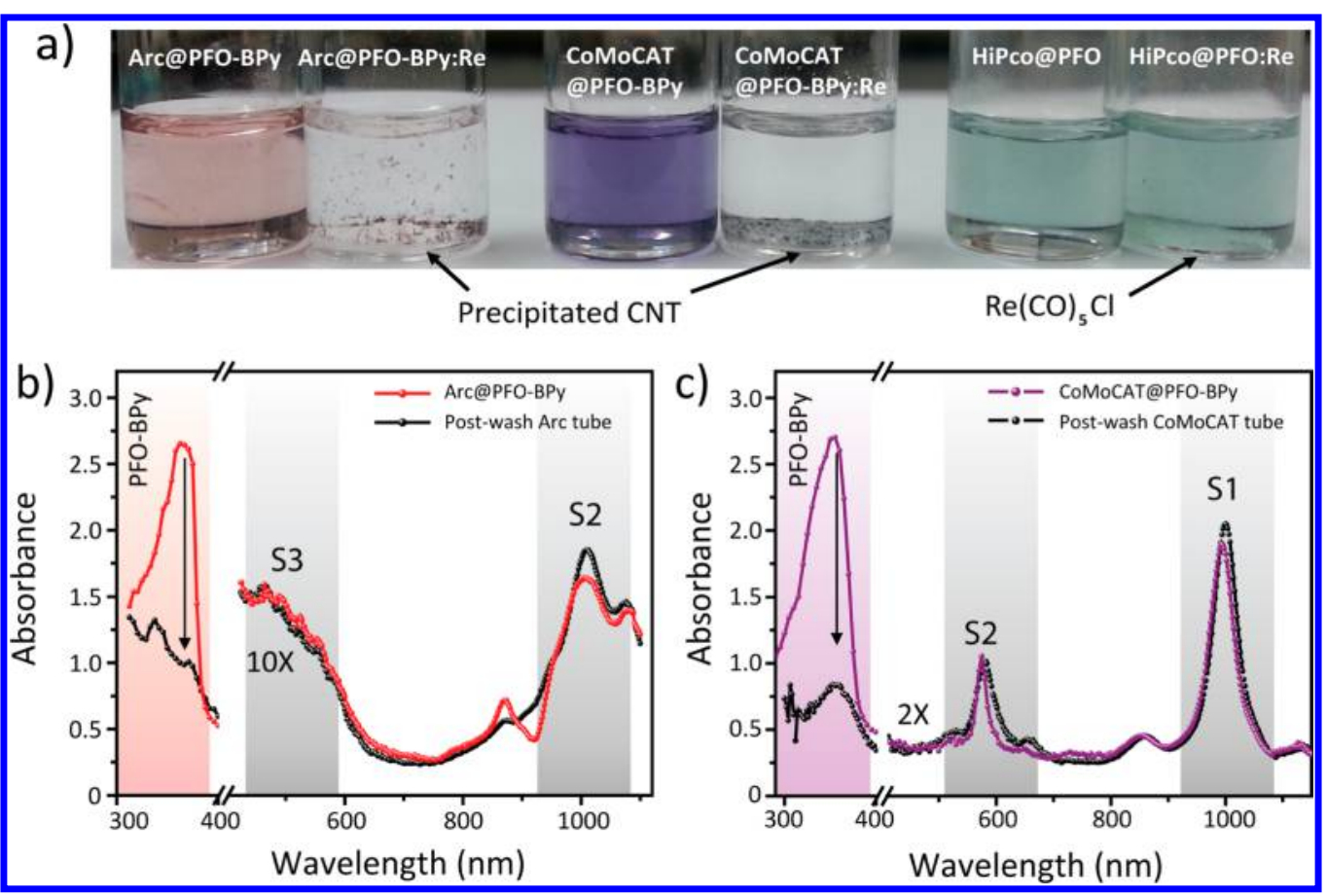

Figure 2. (a) Images of solutions of polymer-wrapped s-SWCNTs before and after the reaction with $\operatorname{Re}(\mathrm{CO})_{5} \mathrm{Cl}$. Images include solutions of Arc@PFO-BPy, post-treatment Arc tubes, CoMoCAT@PFO-BPy, post-treatment CoMoCAT tubes, HiPco@PFO, and post-treatment HiPco tubes. (b) Absorption spectra of Arc@PFO-BPy and postwash Arc tubes. The $\mathrm{S}_{2}$ and $\mathrm{S}_{3}$ optical transitions appear as a group of peaks near 1000 and $500 \mathrm{~nm}$, respectively. The absorbance in the nanotube region is scaled by a factor of 10. (c) Absorption spectra of CoMoCAT@PFO-BPy and postwash CoMoCAT tubes. The $S_{1}$ and $S_{2}$ optical transitions are apparent as sharp peaks at 1000 and $580 \mathrm{~nm}$, respectively. The absorbance in the nanotube region is scaled by a factor of 2 .

in $\mathrm{N}$-cyclohexyl-2-pyrrolidone (CHP) for absorption studies and deposited onto $\mathrm{SiO}_{2} / \mathrm{Si}$ substrates for XPS characterization.

Optical absorption studies for the Arc@ and CoMoCAT@PFO-BPy complexes before and after the addition of $\operatorname{Re}(\mathrm{CO}){ }_{5} \mathrm{Cl}$ confirm the composition of the precipitates and the supernatant solution (Figure 2b,c). The peak corresponding to PFO-BPy, centered at $355 \mathrm{~nm}$, decreased significantly in the postwash tubes. Furthermore, UV-vis absorbance spectrum of the clear supernatant solution (Figure S1, orange curve) is similar to the parent PFO-BPy but with an $\sim 35 \mathrm{~nm}$ red shift in $\lambda_{\text {max }}$. This spectra corresponds to the unwrapped PFO-BPy:Re complex, as metal complexation is known to red-shift the absorbance. ${ }^{25}$ Separately, as a control, we synthesized PFO-BPy:Re by functionalizing PFO$\mathrm{BPy}$ with $\mathrm{Re}(\mathrm{CO}){ }_{5} \mathrm{Cl}$ in the absence of nanotubes and characterized the solution by UV-vis absorption spectroscopy (Figure S1). The UV-vis spectrum of the supernatant solution matches well with that of the synthesized control PFO-BPy:Re complex.

To quantify the removal of PFO-BPy from the SWCNTs, XPS characterization was carried out (Figure 3). The N (1s) peak results exclusively from the PFO-BPy polymer wrap on the SWCNTs. The area under the N (1s) (399.4-402 eV) peak was normalized to that of the $C$ (1s) peak (includes carbon from PFO-BPy wrap and the nanotubes, Figure 3a, Figure S2) and fit with a Voigt function for calculating the reduction ratio $R_{\mathrm{r}}$ defined as $R_{\mathrm{r}}=\left[\left(A_{\mathrm{i}}-A_{\mathrm{f}}\right) / \mathrm{A}_{\mathrm{i}}\right] \times 100$, where $A_{\mathrm{i}}$ and $A_{\mathrm{f}}$ are the areas under the $\mathrm{N}$ (1s) peak before and after $\operatorname{Re}(\mathrm{CO})_{5} \mathrm{Cl}$ treatment. For post-treatment Arc tubes $R_{\mathrm{r}}=50 \%$, which increases to $85 \%$ in the postwash Arc tubes (Figure S2). Any excess uncomplexed $\operatorname{Re}(\mathrm{CO}){ }_{5} \mathrm{Cl}$ on the SWCNTs was also removed in this process as seen by the reduction in the Re (4f) peak (Figure $3 b$ ). In comparison, $R_{\mathrm{r}}$ was only 20\% when the Arc@PFO-BPy was subjected to the same stringent sequential washing steps but without the $\operatorname{Re}(\mathrm{CO})_{5} \mathrm{Cl}$ treatment (Figure 3c, Red curve). This indicates that typically $20 \%$ free PFO-BPy is present in the Arc@PFO-BPy solution, which is removed just by this washing process. For post-treatment CoMoCAT tubes, $R_{\mathrm{r}}=39 \%$, which increases to $71 \%$ in the postwash tubes (Figure S2).

The N (1s) peak from just the polymer PFO-BPy without the tubes was centered at $399.4 \mathrm{eV}$, which shifts to a higher binding energy of $400.8 \mathrm{eV}$ upon complexation with $\operatorname{Re}(\mathrm{CO})_{5} \mathrm{Cl}$. Upon complexation, the PFO-BPy N (1s) peak at $399.4 \mathrm{eV}$ though reduced by $70 \%$ still remains, indicating partial complexation (Figure S3). In the PFO-BPy wrapped tubes, the N (1s) peak (400.8 eV) in Arc@PFO-BPy was downshifted by 0.6 to $398.8 \mathrm{eV}$ compared to just PFO-BPy (Figure 3d). This downshifting is likely due to specific electronic interactions with the nanotubes, which remain undefined. In the post-treatment Arc tubes, three N (1s) peaks were detected at $398.8 \mathrm{eV}$ (red), $400.3 \mathrm{eV}$ (orange), and $402.2 \mathrm{eV}$ (green), which most likely correspond to the nitrogen atoms from BPy units that are 


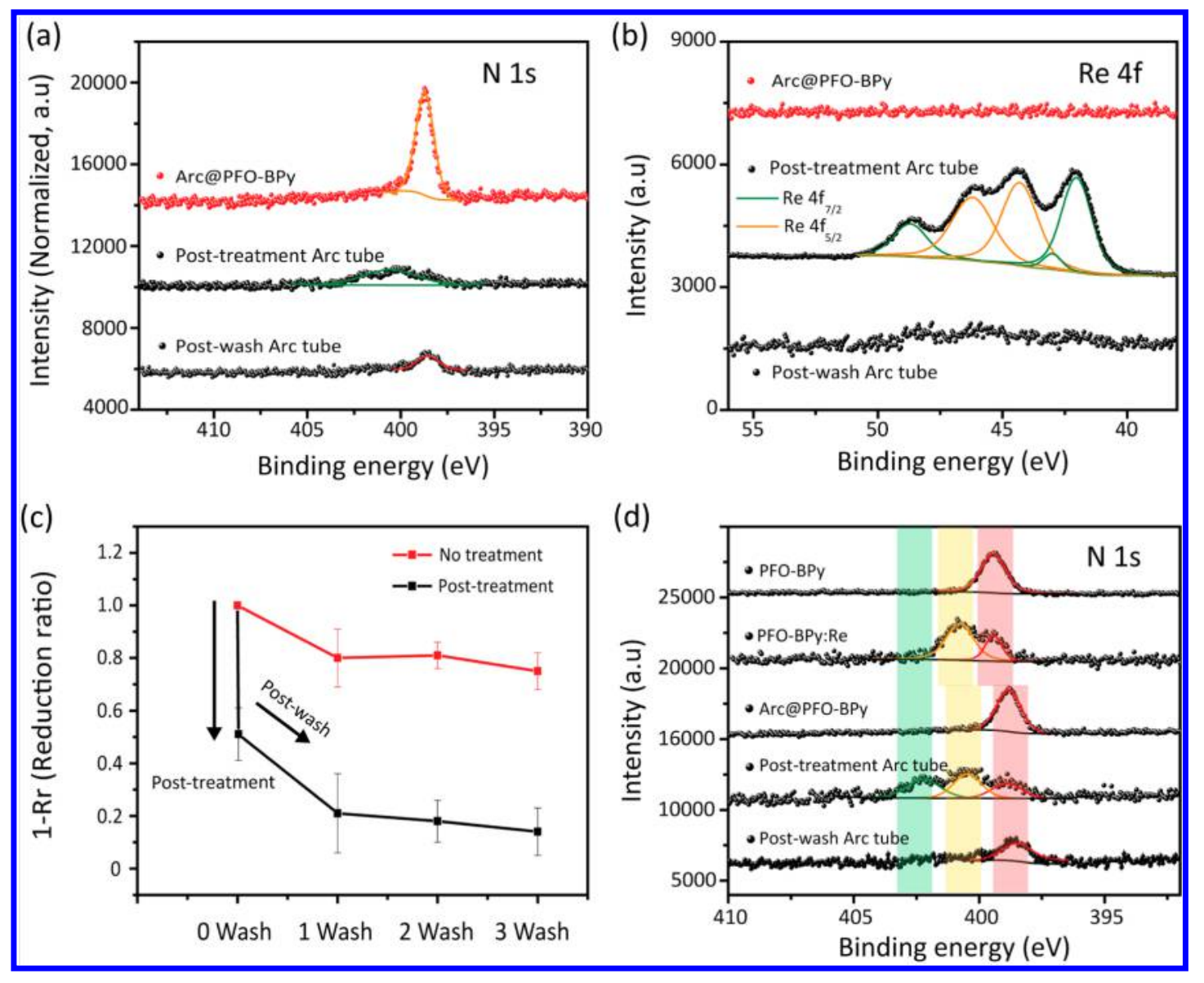

Figure 3. XPS spectra of (a) N (1s) and (b) Re (4f) peaks from Arc@PFO-BPy, post-treatment Arc tubes, and postwash Arc tubes. The peaks are normalized to $C(1 \mathrm{~s})$. (c) Comparison of reduction ratio after additional solvent washing steps with methanol, THF, and chloroform with $\operatorname{Re}(\mathrm{CO})_{5} \mathrm{Cl}$ salt (black line) and without $\operatorname{Re}(\mathrm{CO})_{5} \mathrm{Cl}$ salt (red line) treatment. Data from three wash cycles are plotted. (d) Comparison of the $\mathrm{N}$ (1s) peak from each process (Note unlike panels a, these peaks were not normalized to the $C$ (1s) peak, as the purpose here was to compare peak position, hence the peak intesities were adjusted).

uncomplexed, complexed with $\operatorname{Re}(\mathrm{CO})_{5} \mathrm{Cl}$ but not bound to the nanotubes (from the PFO-BPy:Re unwrapped from the tubes), and complexed with $\operatorname{Re}(\mathrm{CO})_{5} \mathrm{Cl}$ but bound to the tubes, respectively. In the postwash Arc tubes (Figure 3d), the two peaks at 400.3 and $402.2 \mathrm{eV}$ disappeared, confirming the removal of the complexed polymer. The $R_{\mathrm{r}}$ of $85 \%$ deduced from the XPS is in good agreement with the absolute weight loss ( $~ 85 \%)$ of polymer from s-SWCNT, calculated from Thermogravimetric Analysis (TGA) in Figure S4.

The metal complexation route developed here to unwrap the PFO-BPy from s-SWCNTs can be generalized to other metal salts that can effectively chelate with the BPy group and are also soluble in organic solvents (Table 1). Three different metal salts (i) pentacarbonylrhenium chloride $\operatorname{Re}(\mathrm{CO})_{5} \mathrm{Cl}$, (ii) dirheniumdecacarbonyl $\operatorname{Re}_{2}(\mathrm{CO})_{10}$, and (iii) $\mu$-dichlorotetracarbonyldirhodium, $\mathrm{Rh}_{2} \mathrm{Cl}_{2}(\mathrm{CO})_{4}$ were also reacted. The coordinating ability for each of these salts varies leading to changes in $R_{\mathrm{r}}$ from 40 to $85 \%$ as seen from the XPS quantification (Figure S3). Of the complexes, $\operatorname{Re}(\mathrm{CO})_{5} \mathrm{Cl}$ has the most efficient complexation based on the $R_{\mathrm{r}}$ values. This method was also generalizable to a range
TABLE 1. Complexation Ratio of Metal lons $\left(\operatorname{Re}(\mathrm{CO})_{5} \mathrm{Cl}\right.$, $\mathrm{Re}_{2}(\mathrm{CO})_{10}, \mathrm{Rh}_{2}(\mathrm{CO})_{4} \mathrm{Cl}_{2}$ ) with Biypridine Units in PFO-BPy Backbone and the Maximum Reduction Ratio of PFO-BPy from Arc@PFO-BPy and CoMoCAT@PFO-BPy for Different Metal Salts

\begin{tabular}{lccc}
\multicolumn{1}{c}{ metal salts } & $\mathbf{R e}(\mathbf{C O})_{5} \mathbf{C l}$ & $\mathbf{R e}_{2}(\mathbf{C O})_{\mathbf{1 0}}$ & $\mathbf{R h}_{2}(\mathbf{C O})_{4} \mathbf{C l}_{2}$ \\
\hline Complexation Percentage $^{a}$ & $60 \%$ & $55 \%$ & $29 \%$ \\
$\begin{array}{l}\text { Reduction ratio of polymer } \\
\quad \text { from Ar@@PF0-BPy }\end{array}$ & $85 \%$ & $77 \%$ & $52 \%$ \\
$\begin{array}{l}\text { Reduction ratio of polymer } \\
\text { from CoMoCAT@PF0-BPy }\end{array}$ & $71 \%$ & $61 \%$ & $41 \%$ \\
$\quad$ & & \\
\hline
\end{tabular}

${ }^{a}$ Complexation percentage was calculated by comparing the N (1s) peak from PFO-BPy upon complexation with that before complexation with metal salt.

of s-SWCNT diameters from Arc-discharge (1.3-1.7 nm) to smaller CoMoCAT $(6,5)$ nanotubes $(0.7 \mathrm{~nm})$.

We observed several characteristic s-SWCNT peaks in the Raman spectra of Arc@PFO-BPy, namely D-band, and G-band modes, which occur at 1348 and $1605 \mathrm{~cm}^{-1}$, respectively (Figure 4a). ${ }^{26,27}$ The integrated intensity ratio of the $D$ band to the $G$ band, $I_{D} / I_{G}$, is a parameter sensitive to the defect density and doping 


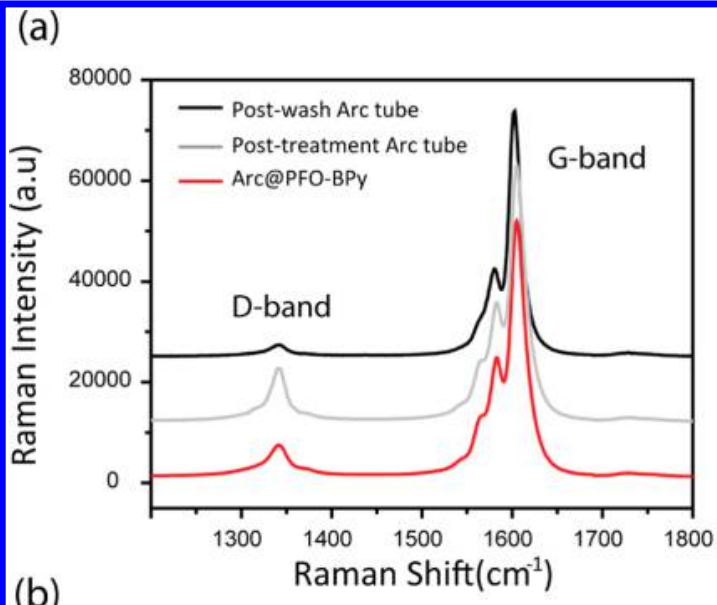

(c)

(b)
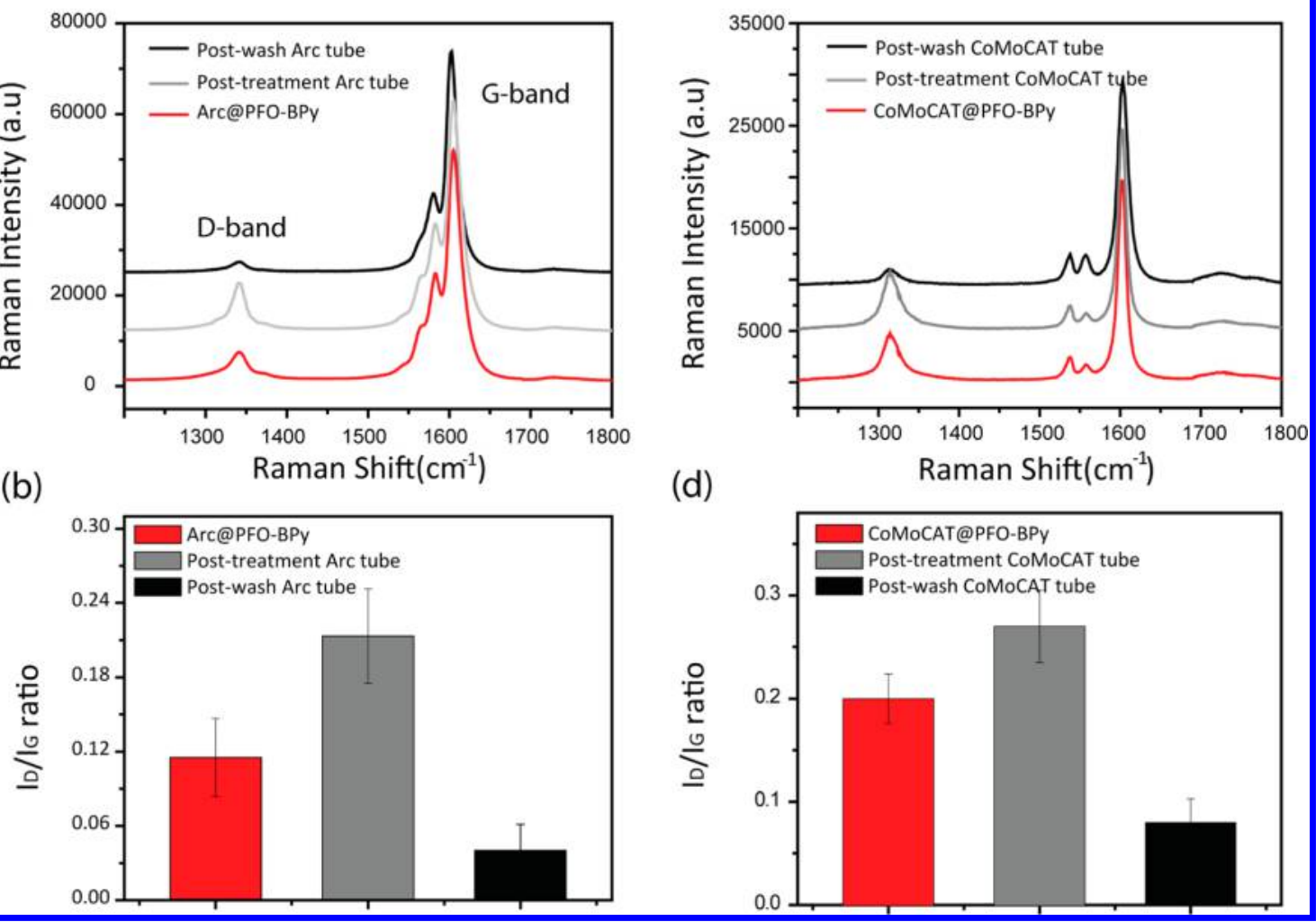

Figure 4. Raman spectra of SWCNT@PFO-BPy, post-treatment, and postwashing tubes, showing Raman G-band, D-band of (a) Arc@PFO-BPy and (c) CoMoCAT@PFO-BPy; $I_{D} / l_{G}$ ratio (integrated intensity ratio of the $D$ band and $G$ band) of (b) Arc@PFOBPy, post-treatment, postwash Arc tube and (d) CoMoCAT@PFO-BPy, post-treatment, and postwash CoMoCAT tubes.

(Figure $4 \mathrm{~b}) .^{28}$ Without $\operatorname{Re}(\mathrm{CO})_{5} \mathrm{Cl}$ treatment, the average $I_{\mathrm{D}} / I_{\mathrm{G}}$ of the Arc@PFO-BPy was 0.12, which increased to 0.21 in the post-treatment Arc tubes, probably due to the presence of excess $\operatorname{Re}(C O)_{5} \mathrm{Cl}$ in the vicinity of the tubes as well as complexation of $\operatorname{Re}(\mathrm{CO}){ }_{5} \mathrm{Cl}$ with PFO-BPy on the s-SWCNT surface. ${ }^{28}$ The $I_{D} / I_{G}$ ratio decreased from 0.21 to 0.05 in the postwash Arc tubes. The effect of surfactant removal on the $I_{D} / I_{G}$ of SWCNTs was previously observed by Kane et al., who attribute fluctuations in $I_{D} / l_{G}$ to changes in doping that result from surfactant removal. ${ }^{29,30}$ These doping effects are apparent in the observed shifts in the 2D bands and radial breathing modes (Figure S5); however, the exact nature of doping is unclear at this point. Moreover, from these results, overall the $I_{\mathrm{D}} / I_{\mathrm{G}}$ ratio stays small throughout the unwrapping reaction, which indicates the defect density is not appreciably increased during the reaction. Similar trends in the $I_{D} / I_{G}$ ratios were observed for CoMoCAT tubes (Figure 4c,d).

The straightforward unwrapping process illustrated in Figure 5 is likely triggered by the conformational change in the polymer due to the formation of a coordination bond. Wang et al. and He et al. have studied the absorption of conjugated polymers upon metal complexation and observed that chelating a metal ion changes the structure of the BPy group from a twisted conformation into a planar structure, ${ }^{31,32}$ thus increasing the rigidity of polymer backbone. In our studies, we find a red shift in PFO-BPy:Re peak $\left(\lambda_{\max }=400 \mathrm{~nm}\right)$ after metal complexation of PFO-BPy $(355 \mathrm{~nm})$, in the UV-vis spectra (Figure 5). This red shift is associated with increased planarity and conjugation. We hypothesize that as more BPy groups in PFO-BPy bind with $\operatorname{Re}(\mathrm{CO})_{5} \mathrm{Cl}$, the increase in rigidity of the polymer backbone spontaneously unwraps the polymer from the s-SWNT surface as depicted in Figure 5 . The $\pi-\pi$ interactions between the PFO-BPy and the nanotubes likely decrease as the rigidity in the chain increases, further contributing to the unwrapping process.

To provide additional theoretical support and test our hypothesis on the rigidity of the PFO-BPy:Re polymer backbone, we carried out large-scale molecular dynamics simulations (see Experimental Section for details) of SWCNTs with the PFO-BPy and PFO-BPy:Re polymers. The MD configurations were initialized with the polymer wrapped around the SWCNT with the oligomers arranged in a helical orientation around the nanotube. The resulting polymer-SWCNT hybrid system was then minimized using the NR/BFGS (Newton-Raphson/Broyden-Fletcher-GoldfarbShanno) method (Figure 6). With these minimized structures, MD simulations were subsequently carried out in the canonical (NVT) ensemble at $300 \mathrm{~K}$. Specifically, we utilized the verlet leapfrog algorithm with the 


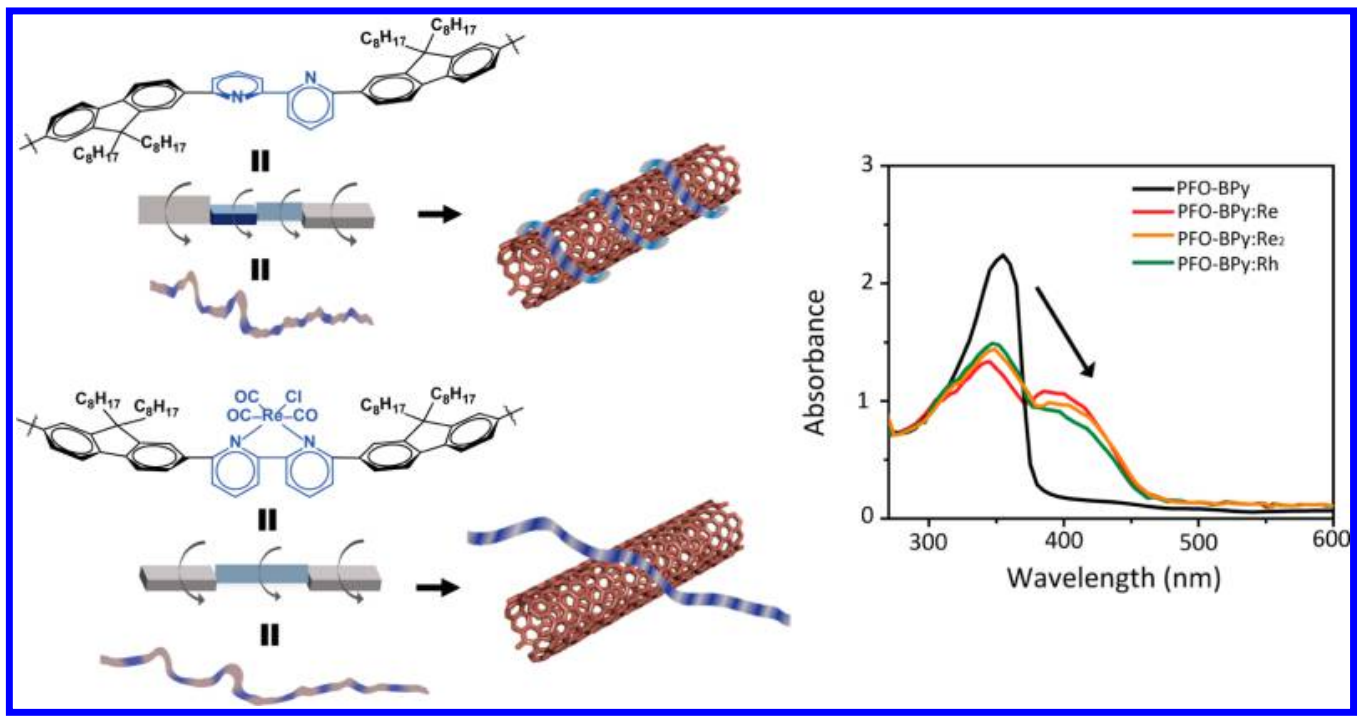

Figure 5. Schematic representation of the envisioned mechanism (left) for stripping of PFO-BPy from s-SWCNTs. Blue and gray regions show the bipyridine and fluorene groups in the PFO-BPy, respectively. (Right) UV-vis absorption spectra of PFOBPy, PFO-BPy:Re, PFO-BPy:Re ${ }_{2}$, and PFO-BPy:Rh show a red shift upon metal complexation.

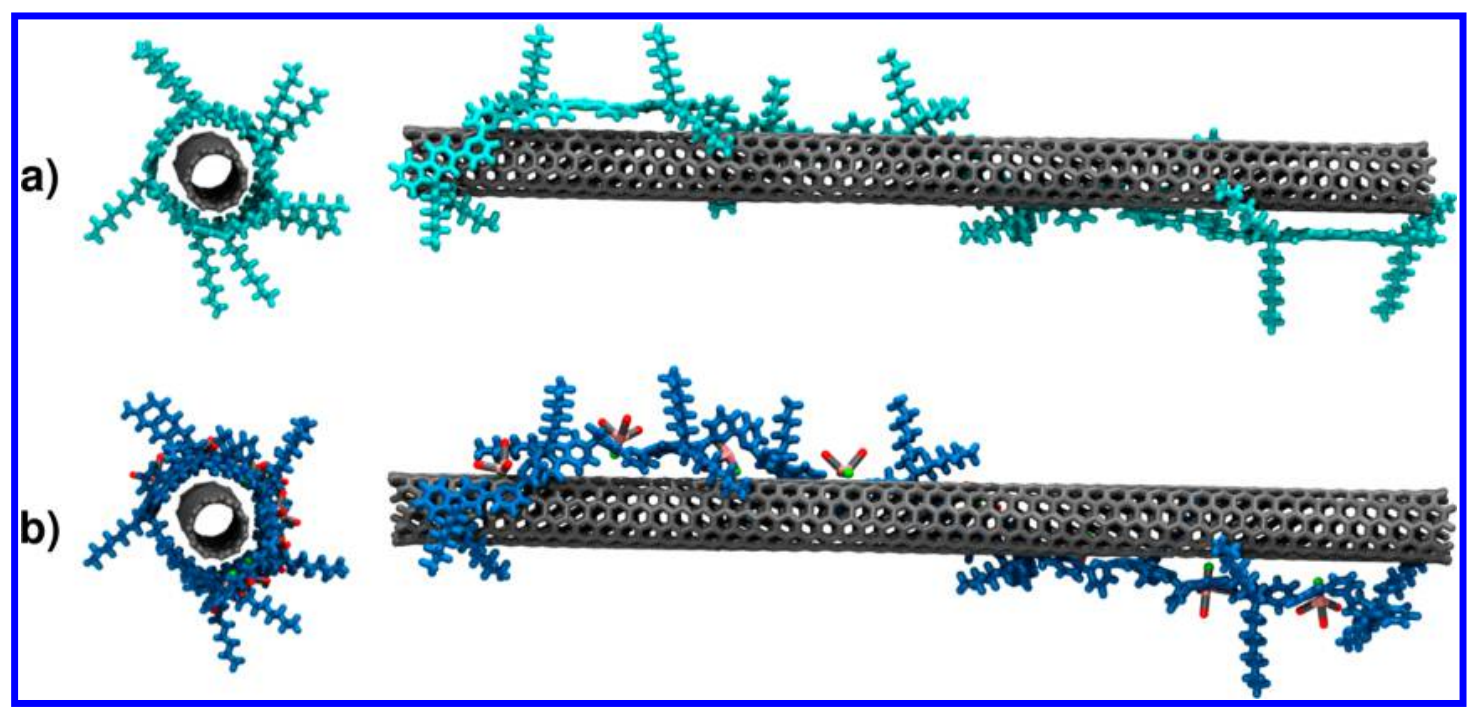

Figure 6. Relaxed MD configurations of a $(6,5)$ SWCNT with the (a) PFO-BPy and (b) PFO-BPy:Re polymers. The figures on the left depict the cross-section of the polymer-SWCNT hybrid structure, and the figures on the right show the overall helical conformation of each polymer around the SWCNT.

Nose-Hoover thermostat for a total time of 100 ps to both eliminate any remaining high-energy structural features and to reach equilibrium. From the equilibrated structures, we performed an additional $2000 \mathrm{ps}$ of dynamics in the NVT ensemble using a time step of $2 \mathrm{fs}$ (see Supporting Information for MD movies). Since we are primarily interested in the adsorption mechanisms of the polymer with the SWCNT, all of the SWCNT atoms were kept fixed during the MD simulations in order to reduce the computation time.

Figure 7 shows selected snapshots of both oligomers along the SWCNT at different time steps during the MD simulations. At $t=0 \mathrm{ps}$, both polymers are clearly seen to be adsorbed and wrapped around the nanotube. However, as we propagate the MD simulations in time, we observe that the PFO-BPy:Re polymer begins to unwrap from the SWCNT, whereas the PFOBPy polymer continues to be wrapped around the SWCNT (see Supporting Information for additional MD movies). These simulations strongly suggest that the chelation of $\operatorname{Re}(\mathrm{CO}){ }_{5} \mathrm{Cl}$ to the PFO-BPy backbone breaks the favorable van der Waals interaction of the benzene rings in PFO-BPy with the SWCNT, and therefore, the interaction between the PFO-BPy:Re polymer with the SWCNT is less favorable. It is worth noting that after the PFO-BPy:Re polymer unwraps from the SWCNT, long-range interactions between the two still exist. The precipitation or phase separation observed in the experiments is, therefore, due to two principal factors that are not taken into account within the simulations. The first factor is the inclusion of other SWCNTs that can affect the separation of the PFO-BPy:Re polymer; 


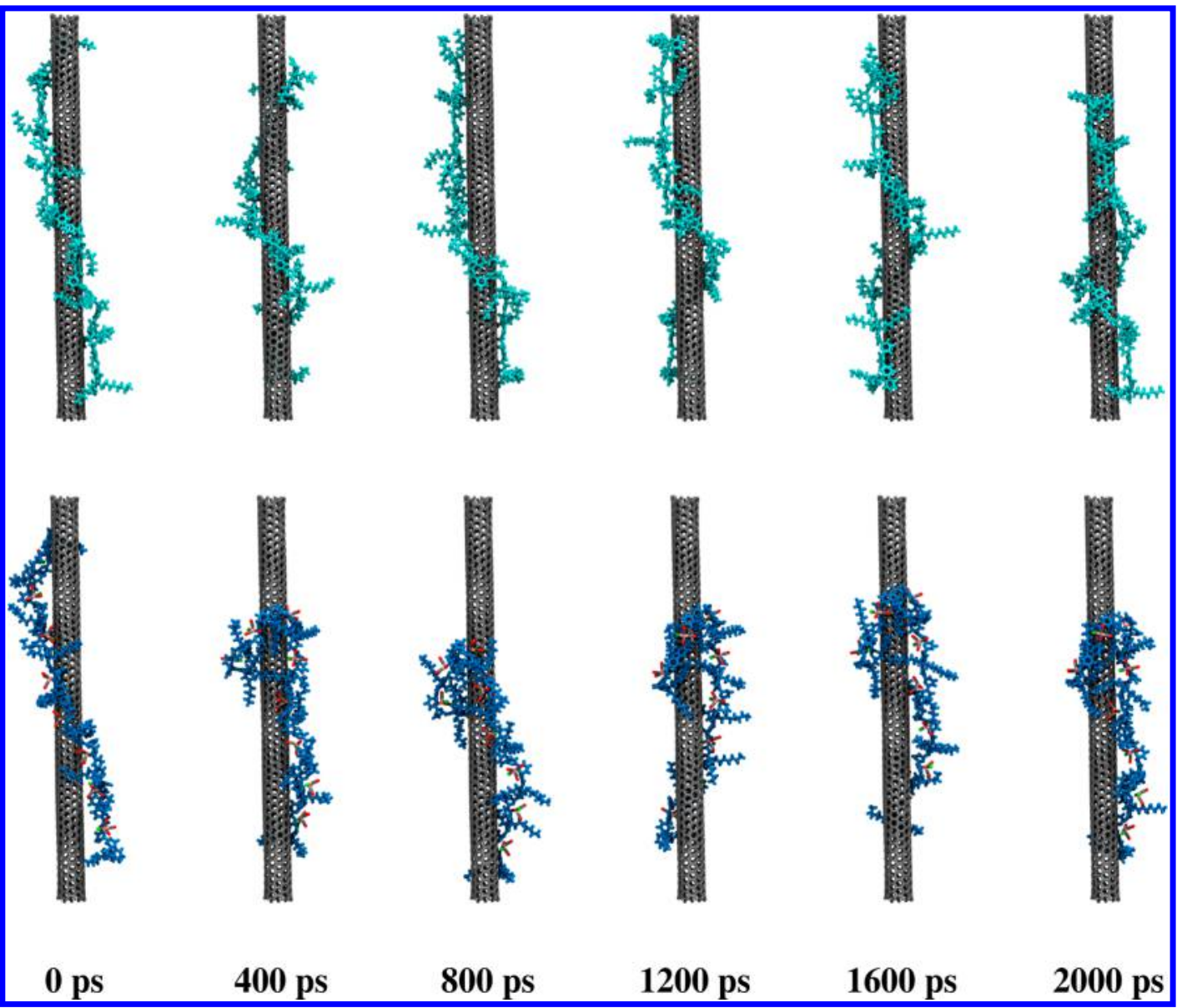

Figure 7. Selected snapshots of PFO-BPy (upper panel) and PFO-BPy:Re (lower panel) along the $(6,5)$ SWCNT as a function of time.

for example, the unwrapping process exposes additional surface area of the SWCNTs leading to additional interactions between the nanotubes. The second factor is the presence and effect of solvent molecules. Our MD simulations did not include solvent effects in order to isolate the dynamics of the oligomers in the proximity of the SWCNTs. It is expected that the inclusion of explicit solvent can favor the unwrapping of the PFOBPy:Re polymer since the SWCNT is more exposed to the solvent molecules leading to a phase separation. ${ }^{33}$ To rigorously quantify the dynamics of both polymer systems, we can numerically evaluate the radius of gyration, $R_{\mathrm{g}}$, given by ${ }^{34-36} R_{\mathrm{g}}^{2}=(1 / N) \sum_{i=1}^{N}\left(r_{\mathrm{i}}-r_{\mathrm{cm}}\right)^{2}$, where $r_{\mathrm{i}}$ and $r_{\mathrm{cm}}$ are the position vectors of each atom and the center of mass of the oligomer, respectively, and $N$ is the number of atoms in the oligomer. The radius of gyration provides a numerical measure of the folding of the polymer chain and its global shape, with higher values of $R_{\mathrm{g}}^{2}$ corresponding to an expanded and stiff molecular configuration.

Figure 8 displays selected MD snapshots of both the PFO-BPy and PFO-BPy:Re polymer chains without the SWCNT. In both cases, we started with an initial configuration corresponding to a linear conformation, and as the MD simulations progress, the PFO-BPy oligomer

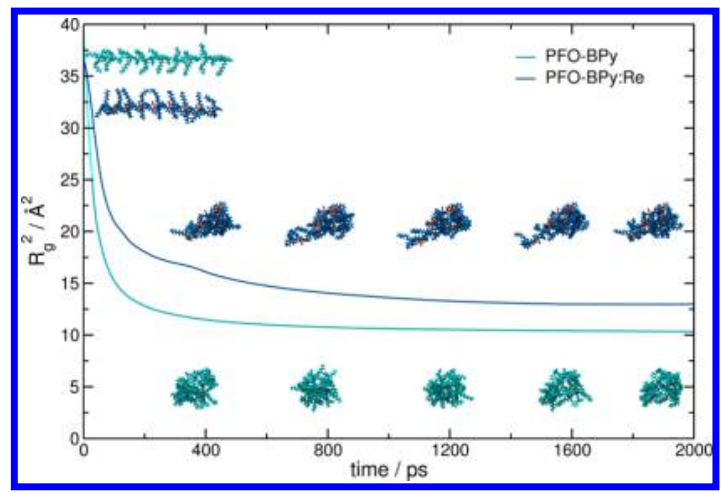

Figure 8. Radius of gyration $\left(R_{\mathrm{g}}\right)$ as a function of time for the PFO-BPy and PFO-BPy:Re polymers. Selected snapshots of PFO-BPy and PFO-BPy:Re as a function of time are displayed inside the graphic.

rapid curls upon itself, whereas the PFO-BPy:Re polymer is more rigid. To rigorously assess the flexibility in each of the oligomers, we also plot the radius of gyration as a function of time in Figure 8. The PFO-BPy has a lower $R_{\mathrm{g}}$ value for all time steps compared to the PFO-BPy:Re oligomer, indicating a loss of flexibility of the molecular chain when the Re complex is present. These results suggest a more favorable interaction between the SWCNT and the PFO-BPy when compared 
with the PFO-BPy:Re polymer. Moreover, these MD simulations confirm our experimental findings that the complexation of $\mathrm{BPy}$ with $\mathrm{Re}(\mathrm{CO})_{5} \mathrm{Cl}$ in the PFOBPy backbone induces a dramatic and irreversible conformational change that leads to a dynamic unwrapping of the entire polymer off the nanotube.

\section{CONCLUSION}

We have demonstrated how a change in polymer backbone rigidity can trigger its release from the nanotube surface. To do so we choose a model polymer, namely PFO-BPy, which is commercially available, that provides ultrahigh selectivity for large-diameter semiconducting carbon nanotubes, which are useful specifically for FETs and has the chemical functionality (BPy) to alter the rigidity using mild chemistry. Postsorting, simple metal complexation leads to the unwrapping of the PFO-BPy from the s-SWNT surface likely due to increased stiffness of the polymer backbone as well as reduced noncovalent interactions with the s-SWCNT surface. The UV-vis, XPS and Raman studies show that the binding of metal ligand complexes to BPy in the PFO-BPy triggers up to $85 \%$ removal of the PFO-BPy from Arc-discharge s-SWCNT (diameter $=1.3-1.7 \mathrm{~nm}$ ) and up to $72 \%$ from CoMoCAT s-SWCNT (diameter $=0.7-0.8 \mathrm{~nm}$ ). In addition, we have performed molecular dynamics simulations to complement our experimental results, and these simulations show that the complexation of BPy with $\mathrm{Re}(\mathrm{CO})_{5} \mathrm{Cl}$ in the PFO-BPy backbone induces conformational changes that lead to a dynamic unwrapping of the polymer off the nanotube. Our method of stripping PFO-BPy from s-SWCNTs post sorting is mild enough to preserve the electronic structure of the tubes as evidenced by retention of the $S_{1}$ and $S_{2}$ transitions as well as retention of the structure as evidenced by low $I_{D} / I_{G}$ ratio. This mild simple process on a widely used PFOBPy polymer for SWCNT sorting is likely to have wide ranging impact in applications for both Arc-discharge and CoMoCAT $(6,5)$ s-SWCNTs for high-performance FET devices and large band gap applications such as photovoltaics. Processing of bare tubes into FET devices is difficult; hence, one can envision redispersing the stripped tubes in a different polymer wrap or small molecule that can play a role in assembling the nanotubes into useful devices, or has specific function in the device itself, and can also be reversibly removed. At a fundamental level, the insight gained by these experiments and simulations can lead to new modified design of existing conjugated polymers to alter the rigidity and hence trigger the nanotube release, by chemical, photochemical or other stimuli.

\section{EXPERIMENTAL SECTION}

Characterizations. UV-vis measurements were performed using a Shimadzu PC-2401 spectrophotometer and home-built setup, in which an input monochromator was used to produce a beam of a wavelength ( $10 \mathrm{~nm}$ resolution) and was used to scan over the range 300-1500 nm. Raman spectroscopy was performed using an Aramis Horiba Jobin Yvon Confocal Raman Microscope $(532 \mathrm{~nm}$ laser excitation wavelength, $6 \mathrm{~W}$ laser power). The laser spot size was $\sim 1 \mu \mathrm{m}^{2}$ and the wavenumber calibration was done using a silicon peak at $520 \mathrm{~cm}^{-1}$.

X-ray Photoelectron Spectroscopy (XPS). A Thermo Scientific K-alpha XPS with microfocused monochromated Al K $\alpha$ X-ray source was used for compositional analysis of the films. The $125 \mathrm{~mm}$ mean radius full $180 \mathrm{deg}$ hemispherical analyzer was operated in constant analyzer energy with $400 \mu \mathrm{m}$ selected area aperture. Survey spectra were collected with pass energy $50 \mathrm{eV}$. The resulting data were analyzed by Avantage software where fully integrated control, acquisition, and peak positioning were characterized by fitting multiplex spectra with Voigt functions.

Sorting of Arc-Discharge CNT. Poly[(9,9-dioctylfluorenyl-2,7diyl)-alt-co-(6,6'-[2,2'-bipyridine])] (PFO-BPy) (American Dye Source, $48 \mathrm{k}$ m.w.) was dissolved in toluene at a concentration of $2 \mathrm{mg} / \mathrm{mL}$ by stirring and heating at $80^{\circ} \mathrm{C}$ until the solution was clear and slightly yellow. Arc discharge soot (Nanolab, Inc.) at a concentration of $2 \mathrm{mg} / \mathrm{mL}$ was dispersed in $50 \mathrm{~mL}$ of PFO-BPy solution using a horn tip sonicator (Fisher Scientific, Sonic Dismembrator 500) at $64 \mathrm{~W}$ power. The sonication time of the initial dispersion was $30 \mathrm{~min}$. Following the initial dispersion, the SWCNT solution was centrifuged (Thermo Scientific, Sorvall WX, swing bucket rotor, $\mathrm{TH}-641$ ) at $300000 \mathrm{~g}$ for $10 \mathrm{~min}$ to remove undispersed material. The upper $90 \%$ of the supernatant was collected and centrifuged for an additional $1 \mathrm{~h}$ at $300000 \mathrm{~g}$. The supernatant was collected and the toluene was distilled, rendering a gel-like PFO-BPy SWCNT mixture, which was then dispersed in tetrahydrofuran (THF). The solution was then iteratively centrifuged and dispersed with bath sonication in THF four times, to rinse off as much excess PFO-BPy as possible. The final solution was prepared by horn-tip sonication of the rinsed SWCNT pellet in chloroform for a total of $1 \mathrm{~min}$.

Sorting of COMoCAT $(6,5)$ CNT. COMOCAT SWCNTs (Southwest Nano Technologies, SG65i) at a concentration of $0.5 \mathrm{mg} / \mathrm{mL}$ were added to the PFO-BPy solution and the mixture was homogenized using the horn-tip sonic dismembrator at $64 \mathrm{~W}$ for $15 \mathrm{~min}$. The solution was centrifuged at $300000 \mathrm{~g}$ for $10 \mathrm{~min}$ to remove aggregates and soot. The supernatant was decanted and filtered through a $5 \mu \mathrm{m}$ filter to further remove aggregates, and immediately rotary evaporated to remove toluene. The remaining sediment was blue-green and was dissolved in approximately $10 \mathrm{~mL}$ of hot chloroform, and diluted to $60 \mathrm{~mL}$ with THF. Then, this solution was centrifuged at $150000 \mathrm{~g}$ for $24 \mathrm{~h}$. The supernatant contains free PFO-BPy, while the SWCNTs settle to the bottom of the centrifuge tube. The pellets were collected, briefly sonicated at low power to redisperse in chloroform, and again diluted with THF and centrifuged. These centrifugation/dispersion steps were repeated 4 times to drive off free PFO-BPy and the final pellet was redispersed in toluene at $10 \mu \mathrm{g} / \mathrm{mL}$.

Complexation Process. To the SWCNT@PFO-BPy solutions $(10 \mu \mathrm{g} / \mathrm{mL}$ ) dissolved in $10 \mathrm{~mL}$ of solvent (either chloroform or toluene for Arc-discharge and CoMoCAT SWCNTs, respectively) was added excess $(10 \mathrm{mg})$ of pentacarbonylrhenium chloride $\left(\operatorname{Re}(\mathrm{CO})_{5} \mathrm{Cl}\right)$, dirhenium decacarbonyl $\left(\operatorname{Re}_{2}(\mathrm{CO})_{10}\right)$, or dichlorotetracarbonyldirhodium $\left(\mathrm{Rh}_{2} \mathrm{Cl}_{2}(\mathrm{CO})_{4}\right)$, and the solution was heated to $60^{\circ} \mathrm{C}$ overnight with stirring. Aggregated dark solid and excess $\mathrm{Re}(\mathrm{CO})_{5} \mathrm{Cl}$ precipitated in the vial. The solution was centrifuged at $10000 \mathrm{~g}$ for $20 \mathrm{~min}$ to remove the aggregates. The supernatant was removed and post-treatment material was collected. To remove the stripped PFO-BPy:Re and excess $\operatorname{Re}(\mathrm{CO}){ }_{5} \mathrm{Cl}$, the precipitate was sequentially washed with chloroform, THF, and methanol three times using bath sonication and centrifugation $(10000 \mathrm{~g}, 20 \mathrm{~min})$. After a washing step with solvent, the bundled s-SWCNTs were redispersed in 
$\mathrm{N}$-cyclohexyl-2-pyrrolidone (CHP) to isolate the s-SWCNTs. After horn-sonication treatment for $5 \mathrm{~min}$, the nanotube suspension was deposited onto $\mathrm{SiO}_{2}(90 \mathrm{~nm}) / \mathrm{Si}$ substrate by drop-casting under vacuum at $60^{\circ} \mathrm{C}$ for $10 \mathrm{~h}$. Dispersed s-SWCNTs deposited on the substrate were used for XPS and Raman characterization.

Molecular Dynamics Simulations. All molecular dynamics simulations were carried out using the Universal Force Field (UFF) ${ }^{37}$ as implemented in GULP code. ${ }^{38}$ All of the initial molecular structures were constructed using the Avogadro code, and a $10 \mathrm{~nm}$ long $(6,5)$ SWCNT (with ends capped with hydrogen atoms to avoid structural distortion due to dangling bonds) was used in all of our simulations. To accurately simulate the largescale interactions with the SWCNT, the PFO-BPy and PFO-BPy:Re polymers were each constructed with 8 repeat units.

Conflict of Interest: The authors declare no competing financial interest.

Supporting Information Available: The Supporting Information is available free of charge on the ACS Publications website at DOI: 10.1021/acsnano.5b03835.

Characterization of binding process with UV-vis absorption and XPS; TGA analysis for calculating the absolute weight loss (PDF)

Movie 1, MD movie of interaction between PFO-BPy and a SWCNT (AVI)

Movie 2, MD movie of interaction between PFO-BPy:Re and a SWCNT (AVI)

Movie 3, MD movie of interaction between PFO-BPy and a SWCNT (AVI)

Movie 4, MD movie of interaction between PFO-BPy:Re and a SWCNT (AVI)

Acknowledgment. This work was supported by the University of Wisconsin-Madison Center of Excellence for Materials Research and Innovation NSF Grant No. DMR-1121288. Partial support is also acknowledged from the National Science Foundation Grant CMMI-1129802 (G.J.B.) and the U.S. Army Research Office, W911NF-12-1-0025 (M.J.S.). G.J.B. also acknowledges support from the National Science Foundation Graduate Research Fellowship Program under Grant No. DGE-1256259. M.B.O. acknowledges support from the Institute for Complex Adaptive Matter for a Postdoctoral Fellowship Award. The University Research Computing Facility at Drexel University is also acknowledged for computing resources.

\section{REFERENCES AND NOTES}

1. Tans, S. J.; Verschueren, A. R. M.; Dekker, C. Room-Temperature Transistor Based on a Single Carbon Nanotube. Nature 1998, 393, 49-52.

2. Kymakis, E.; Amaratunga, G. Single-Wall Carbon Nanotube/conjugated Polymer Photovoltaic Devices. Appl. Phys. Lett. 2002, 80, 112-114.

3. Goldoni, A.; Larciprete, R.; Petaccia, L.; Lizzit, S. Single-Wall Carbon Nanotube Interaction With Gases: Sample Contaminants and Environmental Monitoring. J. Am. Chem. Soc. 2003, 125, 11329-11333.

4. Zheng, M.; Jagota, A.; Semke, E. D.; Diner, B. A.; McLean, R. S.; Lustig, S. R.; Richardson, R. E.; Tassi, N. G. DNA-Assisted Dispersion and Separation of Carbon Nanotubes. Nat. Mater. 2003, 2, 338-342.

5. Arnold, M. S.; Green, A. A.; Hulvat, J. F.; Stupp, S. I.; Hersam, M. C. Sorting Carbon Nanotubes by Electronic Structure Using Density Differentiation. Nat. Nanotechnol. 2006, 1 , 60-65.

6. Engel, M.; Small, J. P.; Steiner, M.; Freitag, M.; Green, A. A.; Hersam, M. C.; Avouris, P. Thin Film Nanotube Transistors Based on Self-Assembled, Aligned, Semiconducting Carbon Nanotube Arrays. ACS Nano 2008, 2, 2445-2452.

7. Liu, H.; Nishide, D.; Tanaka, T.; Kataura, H. Large-Scale Single-Chirality Separation of Single-Wall Carbon Nanotubes by Simple Gel Chromatography. Nat. Commun. 2011, 2, 309.

8. Nish, A.; Hwang, J. Y.; Doig, J.; Nicholas, R. J. Highly Selective Dispersion of Single-Walled Carbon Nanotubes
Using Aromatic Polymers. Nat. Nanotechnol. 2007, 2, 640-6.

9. Brady, G. J.; Joo, Y.; Roy, S. S.; Gopalan, P.; Arnold, M. S. High Performance Transistors via Aligned Polyfluorene-Sorted Carbon Nanotubes. Appl. Phys. Lett. 2014, 104, 083107.

10. Mistry, K. S.; Larsen, B. A.; Blackburn, J. L. High-Yield Dispersions of Large-Diameter Semiconducting SingleWalled Carbon Nanotubes with Tunable Narrow Chirality Distributions. ACS Nano 2013, 7, 2231-2239.

11. Bindl, D. J.; Wu, M.-Y.; Prehn, F. C.; Arnold, M. S. Efficiently Harvesting Excitons from Electronic Type-Controlled Semiconducting Carbon Nanotube Films. Nano Lett. 2011, 11, 455-460.

12. Dowgiallo, A.-M.; Mistry, K. S.; Johnson, J. C.; Blackburn, J. L. Ultrafast Spectroscopic Signature of Charge Transfer between Single-Walled Carbon Nanotubes and $C_{60}$. ACS Nano 2014, 8, 8573-8581.

13. Akazaki, K.; Toshimitsu, F.; Ozawa, H.; Fujigaya, T.; Nakashima, N. Recognition and One-pot Extraction of Rightand Left-Handed Semiconducting Single-Walled Carbon Nanotube Enantiomers Using Fluorene-Binaphthol Chiral Copolymers. J. Am. Chem. Soc. 2012, 134, 12700-12707.

14. Ozawa, H.; Fujigaya, T.; Niidome, Y.; Hotta, N.; Fujiki, M.; Nakashima, N. Rational Concept To Recognize/Extract Single-Walled Carbon Nanotubes with a Specific Chirality. J. Am. Chem. Soc. 2011, 133, 2651-2657.

15. Wang, H. L.; Mei, J. G.; Liu, P.; Schmidt, K.; Jimenez-Oses, G.; Osuna, S.; Fang, L.; Tassone, C. J.; Zoombelt, A. P.; Sokolov, A. N.; Houk, K. N.; Toney, M. F.; Bao, Z. A. Scalable and Selective Dispersion of Semiconducting Arc-Discharged Carbon Nanotubes by Dithiafulvalene/Thiophene Copolymers for Thin Film Transistors. ACS Nano 2013, 7, 26592668.

16. Liang, S.; Zhao, Y.; Adronov, A. Selective and Reversible Noncovalent Functionalization of Single-Walled Carbon Nanotubes by a pH-Responsive Vinylogous Tetrathiafulvalene-Fluorene Copolymer. J. Am. Chem. Soc. 2014, 136, 970-977.

17. Wang, W. Z.; Li, W. F.; Pan, X. Y.; Li, C. M.; Li, L. J.; Mu, Y. G.; Rogers, J. A.; Chan-Park, M. B. Degradable Conjugated Polymers: Synthesis and Applications in Enrichment of Semiconducting Single-Walled Carbon Nanotubes. Adv. Funct. Mater. 2011, 21, 1643-1651.

18. Toshimitsu, F.; Nakashima, N. Semiconducting SingleWalled Carbon Nanotubes Sorting with a Removable Solubilizer Based on Dynamic Supramolecular Coordination Chemistry. Nat. Commun. 2014, 5, 5041.

19. Pochorovski, I.; Wang, H.; Feldblyum, J. I.; Zhang, X.; Antaris, A. L.; Bao, Z. H-Bonded Supramolecular Polymer for the Selective Dispersion and Subsequent Release of LargeDiameter Semiconducting Single-Walled Carbon Nanotubes. J. Am. Chem. Soc. 2015, 137, 4328-4331.

20. Joo, Y.; Brady, G. J.; Arnold, M. S.; Gopalan, P. DoseControlled, Floating Evaporative Self-assembly and Alignment of Semiconducting Carbon Nanotubes from Organic Solvents. Langmuir 2014, 30, 3460-3466.

21. Bindl, D. J.; Shea, M. J.; Arnold, M. S. Enhancing Extraction of Photogenerated Excitons from Semiconducting Carbon Nanotube Films as Photocurrent. Chem. Phys. 2013, 413, 29-34.

22. Brady, G. J.; Joo, Y.; Wu, M.-Y.; Shea, M. J.; Gopalan, P.; Arnold, M. S. Polyfluorene-Sorted, Carbon Nanotube Array Field-Effect Transistors with Increased Current Density and High On/Off Ratio. ACS Nano 2014, 8, 11614-11621.

23. Cao, Q.; Han, S.-J.; Tulevski, G. S.; Franklin, A. D.; Haensch, W. Evaluation of Field-Effect Mobility and Contact Resistance of Transistors that Use Solution-Processed Single-Walled Carbon Nanotubes. ACS Nano 2012, 6, 6471-6477.

24. Franklin, A. D. F.; Damon, B.; Haensch, W. Defining and Overcoming the Contact Resistance Challenge in Scaled Carbon Nanotube Transistors. ACS Nano 2014, 8, 73337339.

25. Zhang, M.; Lu, P.; Wang, X.; He, L.; Xia, H.; Zhang, W.; Yang, B.; Liu, L.; Yang, L.; Yang, M.; Ma, Y.; Feng, J.; Wang, D.; Tamai, N. Synthesis and Photophysical Properties of 
$\pi$-Conjugated Polymers Incorporated with Phosphorescent Rhenium(I) Chromophores in the Backbones. J. Phys. Chem. B 2004, 108, 13185-13190.

26. Dresselhaus, M. S.; Dresselhaus, G.; Saito, R.; Jorio, A. Raman spectroscopy of carbon nanotubes. Phys. Rep. 2005, 409, 47-99.

27. Dresselhaus, M.; Dresselhaus, G.; Jorio, A.; Souza Filho, A.; Saito, R. Raman Spectroscopy on Isolated Single Wall Carbon Nanotubes. Carbon 2002, 40, 2043-2061.

28. Yang, Q.; Shuai, L.; Pan, X. Synthesis of Fluorescent Chitosan and Its Application in Noncovalent Functionalization of Carbon Nanotubes. Biomacromolecules 2008, 9, 34223426.

29. Geng, J.; Kong, B. S.; Yang, S. B.; Youn, S. C.; Park, S.; Joo, T.; Jung, H. T. Effect of SWNT Defects on the Electron Transfer Properties in P3HT/SWNT Hybrid Materials. Adv. Funct. Mater. 2008, 18, 2659-2665.

30. Kane, A. A.; Ford, A. C.; Nissen, A.; Krafcik, K. L.; Leonard, F. Etching of Surfactant from Solution-Processed, TypeSeparated Carbon Nanotubes and Impact on Device Behavior. ACS Nano 2014, 8, 2477-2485.

31. Wang, B.; Wasielewski, M. R. Design and Synthesis of Netal Ion-Recognition-Induced Conjugated Polymers: an Approach to Metal lon Sensory Materials. J. Am. Chem. Soc. 1997, 119, 12-21.

32. He, S.; Buelt, A. A.; Hanley, J. M.; Morgan, B. P.; Tennyson, A. G.; Smith, R. C. Sterically Encumbered Bipyridyl-Derivatized Conjugated Polymers and Metallopolymers Incorporating Phenylenevinylene, Phenyleneethynylene, and Fluorenylene Segments. Macromolecules 2012, 45, 63446352.

33. Tallury, S. S.; Pasquinelli, M. A. Molecular Dynamics Simulations of Flexible Polymer Chains Wrapping Single-Walled Carbon Nanotubes. J. Phys. Chem. B 2010, 114, 4122-4129.

34. Kumar, S.; Pattanayek, S. K.; Pereira, G. G. Organization of Polymer Chains onto Long, Single-Wall Carbon NanoTubes: Effect of Tube Diameter and Cooling Method. $\underline{.}$. Chem. Phys. 2014, 140, 024904.

35. Jeronimo, K.; Cruz, V. L.; Ramos, J.; Vega, J. F.; Trujillo, M.; Muller, A. J.; Martinez-Salazar, J. Computer Simulations of the Early Stages of Crystal Nucleation of Linear and Short Chain Branched Polyethylene on Carbon Nanotubes. Eur. Polvm. J. 2014, 56, 194-204.

36. Rouhi, S.; Alizadeh, Y.; Ansari, R. On the Wrapping of Polyglycolide, Poly(Ethylene Oxide), and Polyketone Polymer Chains Around Single-Walled Carbon Nanotubes Using Molecular Dynamics Simulations. Braz. J. Phys. 2015, 45, 10-18.

37. Rappe, A. K.; Casewit, C. J.; Colwell, K. S.; Goddard, W. A.; Skiff, W. M. UFF, a Full Periodic Table Force Field for Molecular Mechanics and Molecular Dynamics Simulations. J. Am. Chem. Soc. 1992, 114, 10024-10035.

38. Gale, J. D. GULP: A Computer Program for the SymmetryAdapted Simulation of Solids. J. Chem. Soc. Faraday Trans. 1997, 93, 629-637. 